\title{
2967. Deep convolutional neural networks for Bearings failure predictionand temperature correlation
}

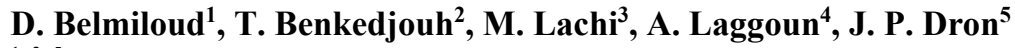 \\ ${ }_{1,3,5}$ GRESPI, LTM Laboratory, Reims Champagne Ardenne University, 51687 Reims, France \\ ${ }^{1}$ LDMV, MI Département, Faculté des Sciences de l'Ingnieur, 35000 Boumerdes, Algeria \\ ${ }^{2}$ LMS Laboratory, EMP, 16111 Algiers, Algeria \\ ${ }^{4}$ UR-MPE Laboratory, Boumerdes University, 35000 Boumerdes, Algeria \\ ${ }^{1}$ Corresponding author \\ E-mail: ${ }^{1}$ dalila.belmiloud@etudiant.univ-reims.fr, ${ }^{2}$ bktarek@gmail.com, ${ }^{3}$ m.lachi@univ-reims.fr, \\ ${ }^{4}$ a_laggoun@yahoo.fr, ${ }^{5}$ jean-paul.dron@univ-reims.fr
}

Received 15 January 2018; received in revised form 2 July 2018; accepted 15 July 2018 DOI https://doi.org/10.21595/jve.2018.19637

Check for updates

Copyright $(C 2018$ D. Belmiloud, et al. This is an open access article distributed under the Creative Commons Attribution License, which permits unrestricted use, distribution, and reproduction in any medium, provided the original work is properly cited.

\begin{abstract}
Rolling elements bearings (REBs) is one of the most sensitive components and the common failure unit in mechanical equipment. Bearings failure prognostics, which aims to achieve an effective way to handle the increasing requirements for higher reliability and in the same time reduce unnecessary costs, has been an area of extensive research. The accurate prediction of bearings Remaining Useful Life (RUL) is indispensable for safe and lifetime-optimized operations. To monitor this vital component and planning repair work, a new intelligent method based on Wavelet Packet Decomposition (WPD) and deep learning networks is proposed in this paper. Firstly, features extraction from WPD used as input data. Secondly, these selected features are fed into deep Convolutional Neural Networks (CNNs) to construct the Health Indicator (HI). This study focuses on analysing the relationships such as correlations between the $\mathrm{HI}$ and temperature. We develop a solution for the Connectiomics contest dataset of bearings under different operating conditions and severity of defects. The performance of the proposed method is verified by four bearing data sets collected from experimental setup called "PRONOSTIA". The results show that the health indicator obtains fairly high monotonicity and correlation values and it is beneficial to bearing life prediction. In addition, it is experimentally demonstrated that the proposed method is able to achieve better performance than a traditional neural network based method.
\end{abstract}

Keywords: bearing, WPD, features extraction, CNNs, prognostic, RUL, temperature.

\section{Introduction}

The performance degradation assessment of bearing plays an important role in various rotating machines fault to guarantee reliability in industrial processes [1-4]. Recently, studies on bearings prognostics can be seen as a problem of pattern recognition and many artificial intelligent methods, several researches have been made to develop techniques for machine health monitoring [5-7].

Cerrada et al. [8] gives a summary of PHM tools for bearings severity evaluation and possible failure modes, characteristics, the common available data types from different sensors, the different features and algorithms applied for prognostics and health management design.

Machine learning algorithms used in health assessment and RUL estimation depend on the features extracted. Recent developments of prognostics have focused on applying advanced techniques of signal processing to extract the robust features for constructing the health indicator [9]. Features extraction from different signal representation such as: temporal, spectral and time-frequency $[10,11]$, and so on, in general do not have good monotonicity, which limits their indication in faults severity evaluation. The features extracted from sensors signals contain information about the health state of bearing. In order to produce robust and significant features, diverse original features need to be studied for show the effectiveness of the proposed approach.

Several researches have been studied the feasibility of ANN for health assessment RUL 
estimation. However, its accuracy is highly dependent on the neural networks structure such as a number of hidden layers, nodes and kernel function. BenAli et al. [12] proposed an intelligent method based on the data-driven prognostic approach by the combination of the neural networks and Weibull distribution. Rai et al. [13] combined the neural network approach with wavelet-based denoising method for the RUL assessment. For the improvement of the traditional neural network, CNNs have been proposed in this study for learn the features, a long short term memory based neural networks scheme was proposed by Yuan et al. [14] utilizing Long Short-Term Memory neural network to get good diagnosis and prediction performance in the cases of complicated operations, hybrid faults and strong noises for RUL estimation of aero-engines in the cases of complicated operations, hybrid faults and strong noises. However, it still to develop an effective approach based on historical data such as deep learning.

Recently, several learning methods has emerged called as deep learning that improved to learn higher level abstractions from the raw data [15-17], deep learning models automatically learn a feature representation from raw signal. CNNs, auto-encoders and deep belief network are the mostly known models in deep learning, and applied in many research area such as: speech recognition [18], image processing [19, 20], machinery condition monitoring and health assessment [21-24]. Li et al. [25] propose a novel deep convolutional neural network-based method for remaining useful life predictions. The aim of this study is to estimate the RUL of aero-engine units accurately. A good prognostic performance prediction is achieved with the proposed approach using raw feature selection, data pre-processing and sample preparation with time window.

In this paper, we propose a model for health assessment and RUL estimation which integrates a WPD algorithm for fast feature extraction, a nodes energy features selection of each wavelet level decomposition, and an intelligent analysis method based on the CNNs algorithm to obtain the health assessment result of bearings. An experimental verification is given by using the data from PRONOSTIA test rig [26]. Then obtained model is applied to the acceleration signals that are collected from the bearing degradation test experiment.

This paper starts with the description of the prognostics and health management along with the proposed model in Section 2. A brief introduction of WPD for features extractions is presented in Section 3 and deep CNNs for bearing health assessment in Section 4. The proposed method is experimentally validated using bearing's dataset in Section 5 and we demonstrated the effectiveness of the proposed approach. We close the paper with conclusions in Section 6.

\section{The proposed model}

PHM is a new concept paradigm of condition based maintenance for The improvement of system safety and reliability by monitoring the facility conditions, including the maximum of the operational availability and reduction of maintenance costs. In general, the PHM methods can be grouped in three main categories such as: data-driven approaches, model-based approaches and statistical approaches $[4,27,2]$. The PHM technologies are evolving rapidly in recent years due to the different statuses and requirements of the cases, the project involved research and development. In Fig. 1 several methods and technologies which can be regarded as the steps towards prognostics and identifying maintenance needs, to support decision making and manage operational reliability. The performance assessment lean model generally consists of three main aspects: health indicators construction, RUL estimation, and health management [2].

Fig. 1 illustrates the overall structure of a typical bearing PHM system. It consists of essential modules, namely, sensing, preprocessing, feature identification, and PHM. The challenge features selection for identifying bearing failure are important step in PHM and inspired great research interest. The PHM module of bearing systems in general start by anomaly detection, fault diagnosis, prognosis, and decision-making in the final step.

The failure threshold limited by using the international standards (ISO 13381-1, ISO 10816 and ISO 7919). The ISO standards limited in vibration signals energy (the root mean square RMS 
of vibration signal), and for different indicators are used [28]. An illustration of RUL progression is shown in Fig. 2.

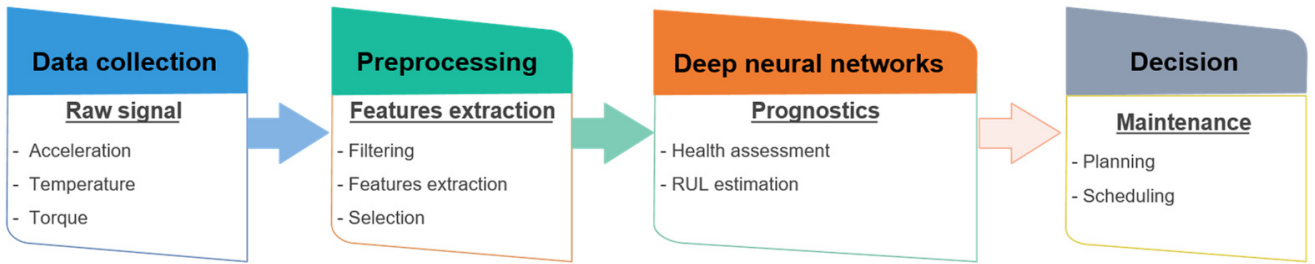

Fig. 1. The flowchart of the lean model for performance assessment

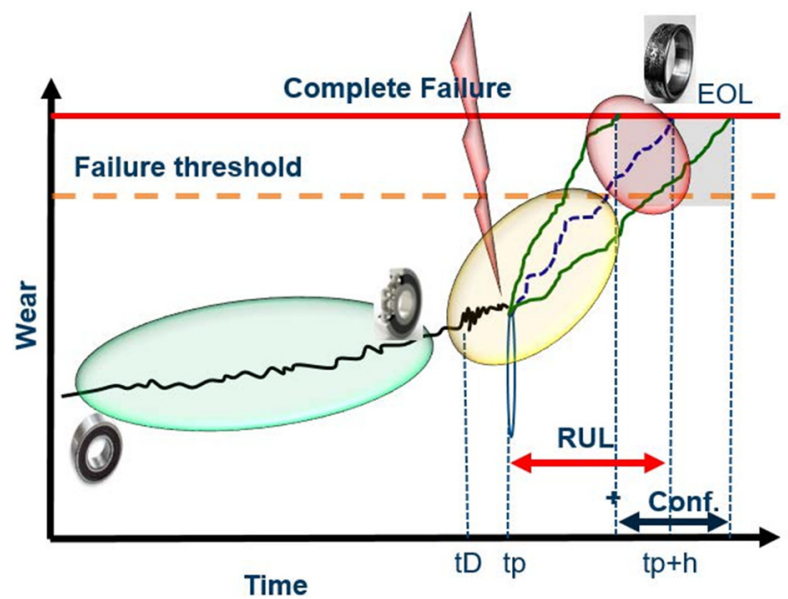

Fig. 2. Illustration of remaining useful life

\section{Principle of WPD for feature extraction}

In the proposed approach firstly, raw signals are processed by a fast, memory-saving algorithm, that is the Wavelet packet decomposition WPD nodes energy of each level is calculated. WPD is a natural extension of Multi Resolution Analysis (MRA) technique [29]. WPD decomposes the signal using both low-frequency components and high-frequency components (Fig. 3); The flexibility of collection of abundant information for the extraction of features that combine non-stationary and stationary characteristics [30]. The multi-level filtering process or decomposition process shown in Fig. 3.

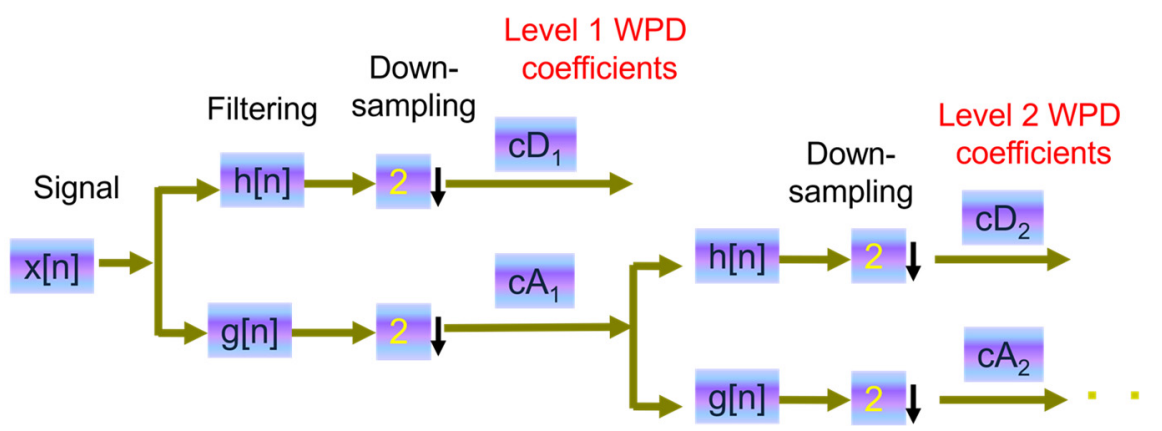

Fig. 3. Structure of two layers wavelet packet decomposition

The discrete signal is convolved with a low pass filter $(\mathrm{g})$ and a high pass filter $(\mathrm{h})$ resulting in two vectors $c A$ called the approximation coefficients and $c D$ called the details coefficients. 
The process of decomposition can be repeated on the approximation vector $c A_{1}$ and successively on every new approximation vector $c A_{i}$. This concept is presented by means of a wavelet tree having $i$ levels, where $i$ is the number of iterations of the basic step. In Fig. 3 the level of decomposition equal 2.

In the current contribution, the original features are extracted from raw signals related to bearings degradations shown in the Table 1.

Table 1. Feature extraction

\begin{tabular}{|c|c|}
\hline Approximation coefficients energy (5 levels (db6)) & $R M S=\sqrt{\frac{1}{N} \sum_{i=1}^{N} c A(t)_{i}^{2}}$ \\
\hline Detail coefficients energy (5 levels (db6)) & $R M S=\sqrt{\frac{1}{N} \sum_{i=1}^{N} c D(t)_{i}^{2}}$ \\
\hline Signal to noise ratio (SNR) & $S N R=\frac{P_{\text {signal }}}{P_{\text {noise }}}$ \\
\hline Peak signal to noise ratio (PSNR) & $P S N R=10 \cdot \log \left(\frac{M A X_{x}^{2}}{M S E}\right)$ \\
\hline
\end{tabular}

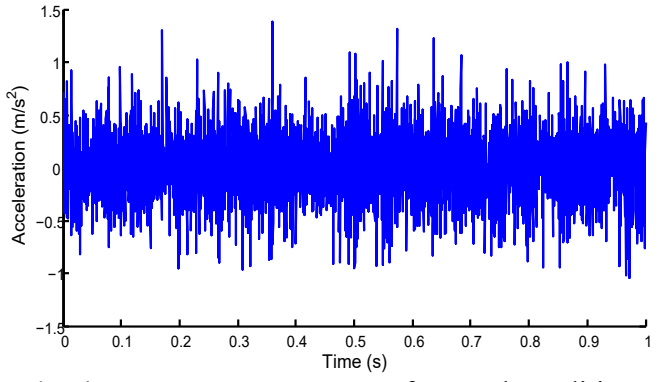

Fig. 4. Sensors measurement of normal conditions

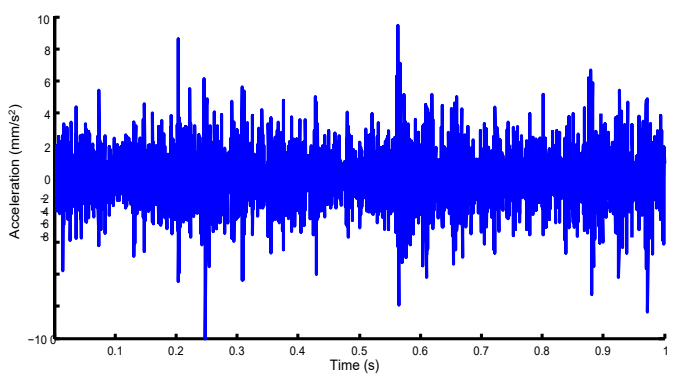

Fig. 5. Sensors measurement degraded bearing

In Fig. 6 shown that there are a few helpful to consider when looking at the multitude of time frequency transform. It is helpful to go over a couple of these with another statistical parameters, because they are heavily utilized in condition monitoring. Increasing sample rate does not add information to frequency peaks of interest. The nodes energy coefficients evolution at each level shown in 7 is equal to the length of raw signals after decomposition using (db6), the WPD coefficients can retain more fault severity information, and hence can be extracted more distinguishing statistical features for RUL estimation.

In Fig. 8 shown that the monitoring temperature on the surface of the bearing housings with temperature probe (PT100) has been used in this paper. Under different operating conditions of load and speed, the temperature of a bearing is monitored for changes that can indicate defect in bearing elements. In this study the experimental has shown that temperature is a good indicator of load, speed or lubrication than of bearing condition.

Temperature monitoring techniques may be helpful in preventing machine breakdown. However, bearing defects have not been found to cause an appreciable increase in temperature until the damage has reached a severe state.

In Fig. 8 shown that the monitoring temperature technique for bearing prognostics becomes far more complicated when it is subject to changeable operating condition. In most situations, varying operating conditions refer to variable loading conditions since it is the major source of contribution to the energy of the measured vibration signal. 


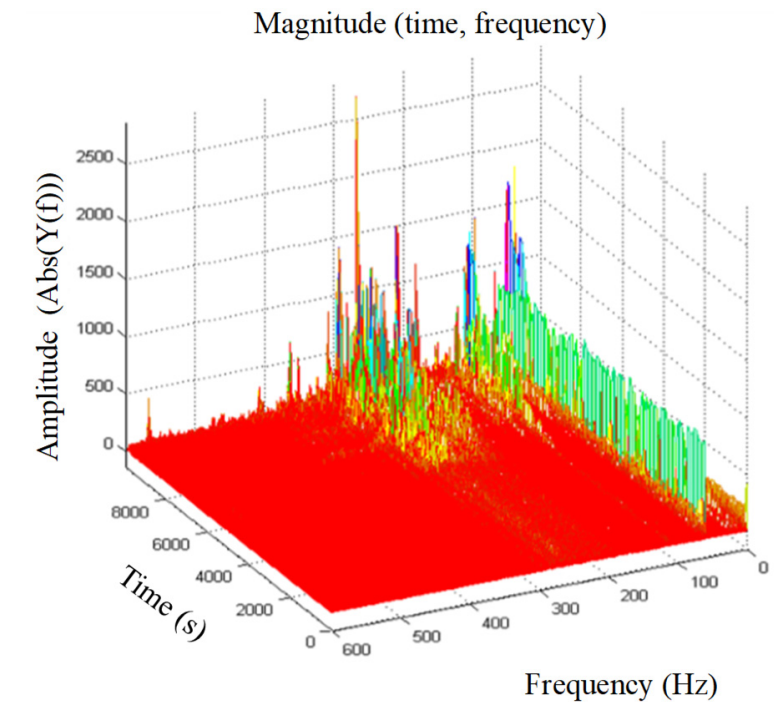

Fig. 6. Time frequency transform for tested bearing $(6 \mathrm{~h} 50)$

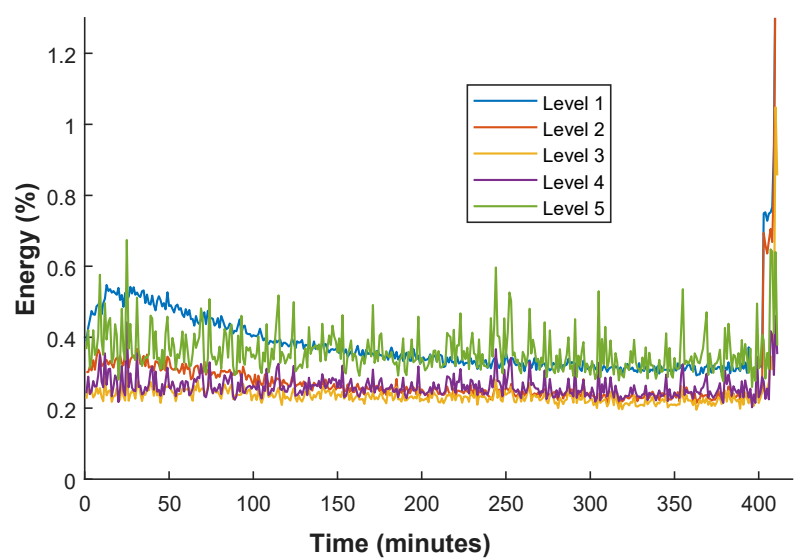

Fig. 7. Wavelet packet decomposition for tested bearing (6h50)

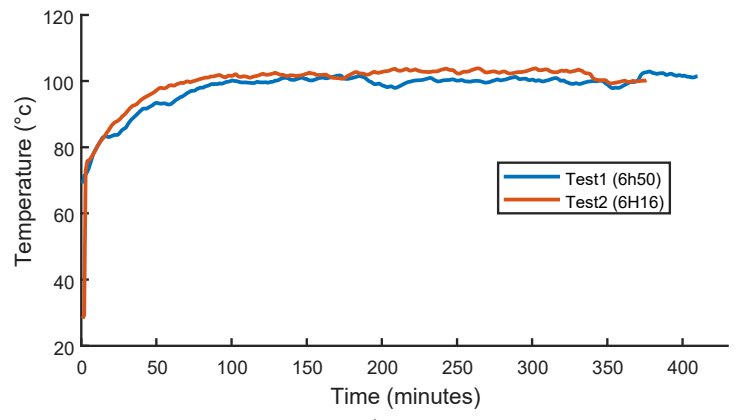

a)

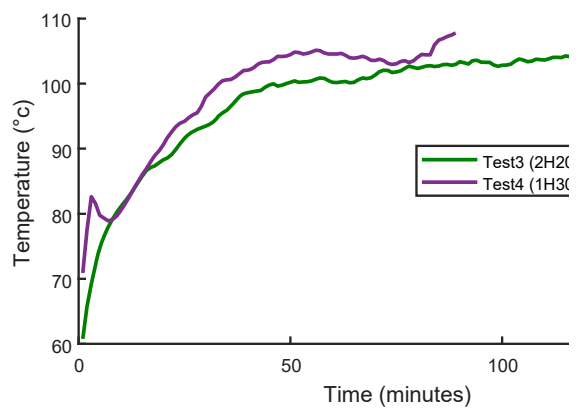

b)

Fig. 8. Temperature evolution for tested bearing

\section{Deep CNNs-Based bearings health assessment}

This section presents the architecture of deep learning CNNs for health assessment and RUL estimation from different sensor signals. The inputs features are extracted from different operating 
condition history. The output values are the RUL of bearings at corresponding lifetime. In honor Yann LeCun [31] an early pioneer of CNNs and the first to reduced them to practice by training them with gradient descent. For image processing, which is featured by two key properties: spatially shared weights and spatial pooling.

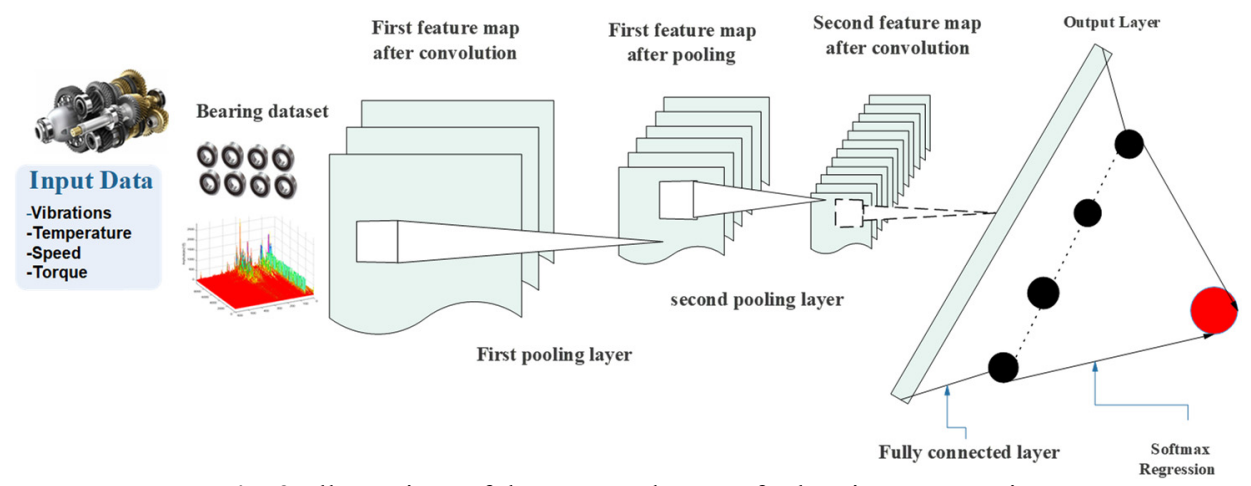

Fig. 9. Illustrations of the proposed CNNs for bearing prognostics

The deep learning algorithms are machine learning technique based on distributed representations. Using structures composed of multiple non-linear transformations deep learning attempts to learn high-level features in data. The frequently used models are CNNs and Deep Belief Network (DBN).

CNNs is also a type of feedforward neural network which is composed of alternating convolutional and subsampling layer $[32,33]$. CNNs are designed to use minimal amounts of preprocessing, which is the main difference compared to other deep architectures.

Firstly, we assume that the input sequential data is $y=\left[y_{1}, \ldots y_{n}\right]$ that $n$ is the length of the sequence and $y_{i} \in R^{d}$ at each time step. Convolution: the dot product between a filter vector $u \in R^{m} d$ and an concatenation vector representation $y_{i: i+m-1}$ defines the convolution operation as follows:

$c_{i}=\varphi\left(u^{T} y_{i: i+m-1}+b\right)$,

where $b$ and $\varphi$ denotes bias term and non-linear activation function, respectively. $y_{i: i+m-1}$ is a $m$-length window starting from the $i$ th time step, which is described as:

$y_{i: i+m-1}=y_{i} \oplus y_{i+1} \oplus \ldots \oplus y_{i+m-1}$.

As defined in Eq. (2), the output scale $c_{i}$ can be regarded as the activation of the filter $u$ on the corresponding subsequence $y_{i: i+m-1}$. By sliding the filtering window from the beginning time step to the ending time step, a feature map as a vector can be given as follows:

$c_{j}=\left[c_{1}, c_{2}, \ldots, c_{l-m+1}\right]$,

where the index $j$ represents the $j$ th filter. It corresponds to multi-windows as:

$\left\{y_{1: m}, x_{2: m+1}, \ldots, y_{l-m+1: l}\right\}$. Max-pooling: able to reduce the length of the feature map, which can minimize the number of model parameters. The hyper-parameter of pooling layer is pooling length denoted as $s$. MAX operation is taking a max over the s consecutive values in feature map $c_{j}$.

Then, the compressed feature vector can be obtained as:

$h=\left[h_{1}, h_{2}, \ldots, \frac{h_{l-m}}{s}+1\right]$, 
where $h_{j}=\max \left(c_{(j-1) s}, c_{(j-1) s+1}, \ldots, c_{(j s-1}\right)$. Then, via the two layers: convolution and max-pooling ones, the fully connected layers and a softmax are usually added to make predictions by the top layers.

The proposed method diagram for the bearings health assessment and RUL estimation shown in Fig. 10. The method is decomposed into two main phases the preprocessing data and training data using deep CNNs. the bearing dataset prepared for the training by computing the features. All the layers use activation functions tan $h$. Then training and testing datasets are prepared for deep CNNs. In the second phase, which is achieved on-line, deals with the utilization of the model generated continuously to assess the health state of the bearing and RUL prediction.

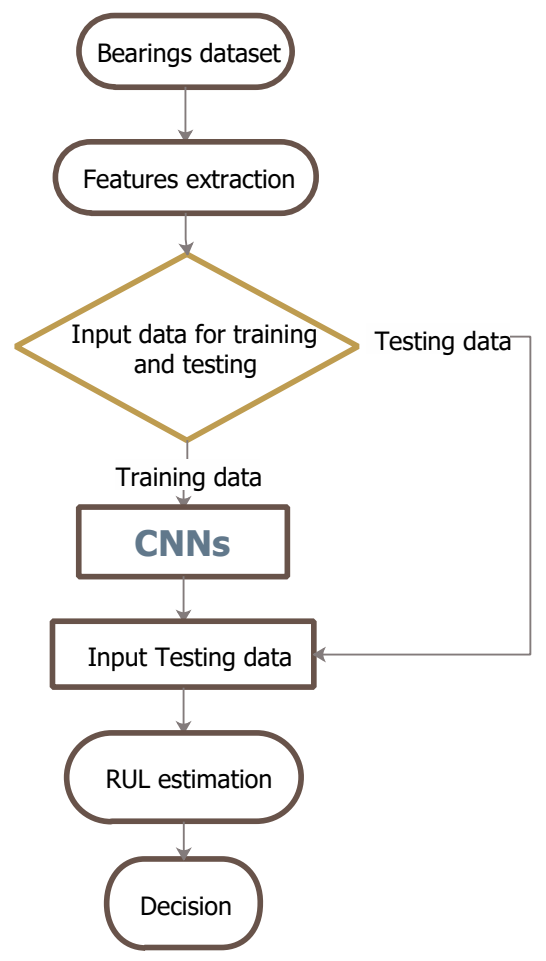

Fig. 10. Flow chart of the proposed method

\section{Application: performance assessment of REBs}

\subsection{Experimental system}

An accelerated bearing life test platform called PRONOSTIA [26] (Fig. 11) is used in this section to verify the prognostics of proposed method. PRONOSTIA is a laboratory experimental platform dedicated to test, verify and validate developed methods related to bearing health assessment, diagnostic and prognostic. In the following of the paper, a set of four experiments consisting of four degraded bearings has been utilized. The four data shown in Table 2 for the different loading and speed motor.

Table 2. Bearings dataset from PRONOSTIA experimental setup

\begin{tabular}{|c|c|c|}
\hline Tests duration & Loading (N) & Speed (RPM) \\
\hline h50 & 4000 & 1800 \\
\hline h16 & 4000 & 1800 \\
\hline h20 & 6000 & 1500 \\
\hline h30 & 8000 & 1500 \\
\hline
\end{tabular}




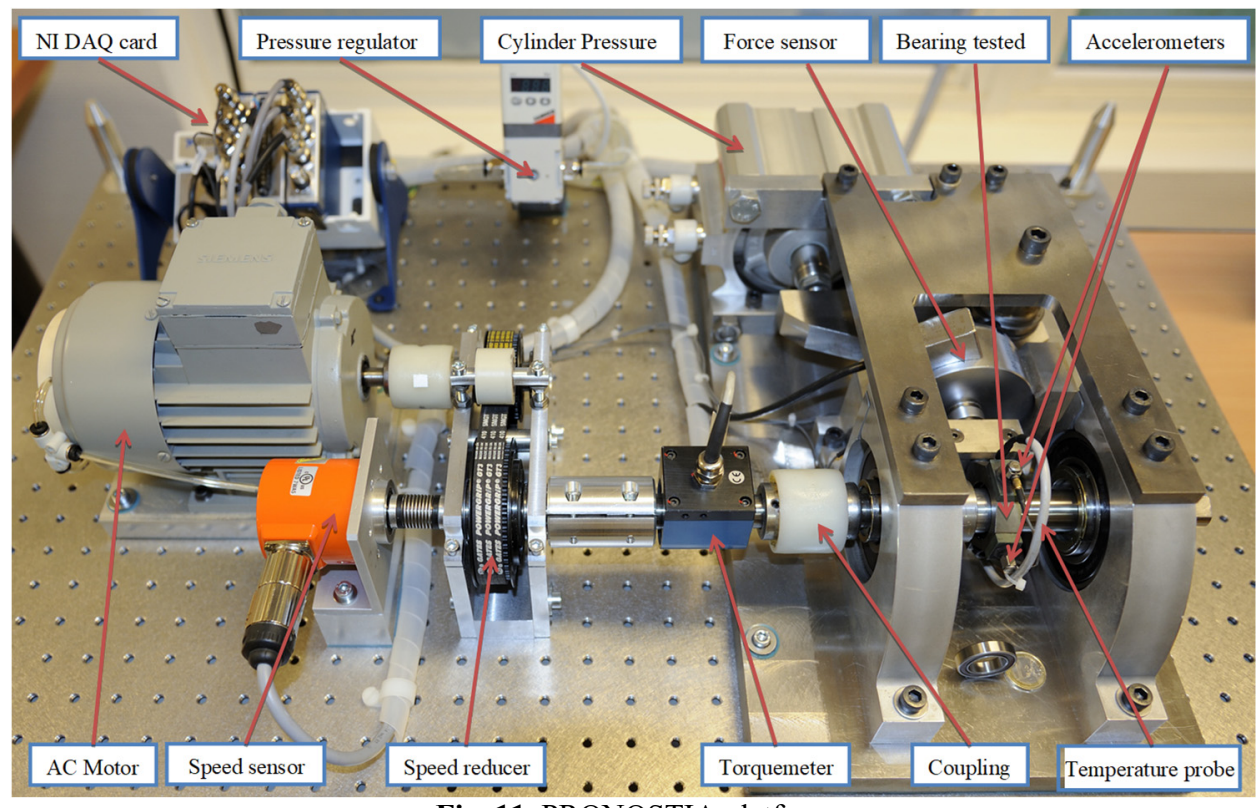

Fig. 11. PRONOSTIA platform

The PRONOSTIA experimental setup composed of two main parts: The first part related to the speed control and a second part to load profiles generation. The speed control part is composed of electric motor, shaft, a set of bearings. The power developed by motor equal to $1.2 \mathrm{~kW}$ and its speed varies between 0 to $6000 \mathrm{rpm}$. The second part of PRONOSTIA contain a hydraulic jack connected to a lever arm used to create different loads on the tested bearing mounted on the platform.

A pair of ball bearings is mounted on one end of the shaft to serve as the guide bearings and a NSK6307DU roller ball bearing is mounted on the other end to serve as the test bearing. The transmission of the movement between the motor and the shaft drive is done by a rub belt. Two accelerometers (DYTRAN3035B) mounted horizontally and vertically on the housing of the tested bearing to pick up the horizontal and the vertical accelerations (Table 3). In addition, the monitoring system includes one temperature probe and a torque sensor (Fig. 11). The sensors are connected to a data acquisition card.

The data acquisition software is programmed by using a LabView interface. Each record is stored in a matrix format where the following parameters are defined: the time, the horizontal acceleration, the vertical acceleration, the temperature, the speed and the torque.

Table 3. Experimental data acquisition

\begin{tabular}{|c|c|}
\hline Measurement & Type \\
\hline Acceleration & (DYTRAN3035B) \\
\hline Temperature & PT100 \\
\hline Torque & (DR2269) \\
\hline Force & (AEPC2S) \\
\hline Acquisition card & (NIDAQCard-9174) \\
\hline Sampling frequency & $25600 \mathrm{~Hz}$ \\
\hline Motor speed & $6000 \mathrm{rpm}, 1.2 \mathrm{~kW}$ \\
\hline
\end{tabular}

With this experimental platform, several types of profile can be created by varying the operating conditions (speed and load). The bearing's behavior is captured during its whole degradation process by using the dedicated sensors. The tested bearing has the characteristics shown in Table 4. 
Table 4. Tested bearing

\begin{tabular}{|c|c|c|c|c|c|}
\hline Bearing type & $\varnothing$ Outside & $\varnothing$ Inside & Balls & Angle & $\varnothing$ Ball \\
\hline NSK6804DD & $32 \mathrm{~mm}$ & $20 \mathrm{~mm}$ & 8 & $\alpha=15^{\circ}$ & $3.5 \mathrm{~mm}$ \\
\hline
\end{tabular}

\subsection{Results and discussion}

In order to identify different working conditions monitoring of REBs, the proposed fault prognostics method based on CNNs and WPD is performed. The initial operator $P$ and $U$ with $M=4$ and $N=4$ are calculated. In this experimentation, each signal was decomposed to level 6 , then eight subband coefficients were obtained. The classification errors are reported in Table 5. The PRONOSTIA experimental setup system are shown in Fig. 11. A working cycle of vibration for different loading and speed motor are saved, which include all the data for each REBs. The classification accuracies of REBs on the PRONOSTIA experimental setup were calculated and reported in Table 5. Also, the classification results obtained by using the features extracted from time domain (Fig. 4 and 5) and from time-frequency domain (Fig. 6).

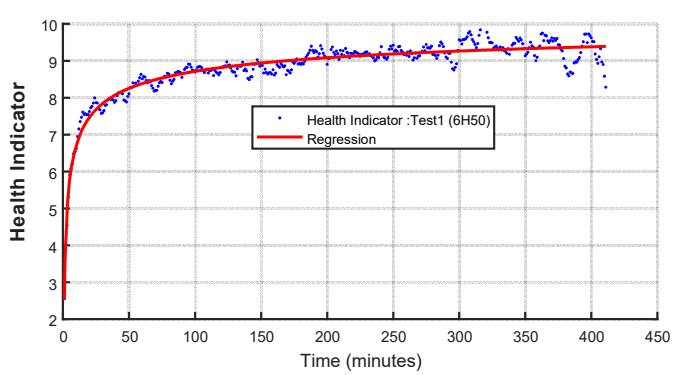

a)

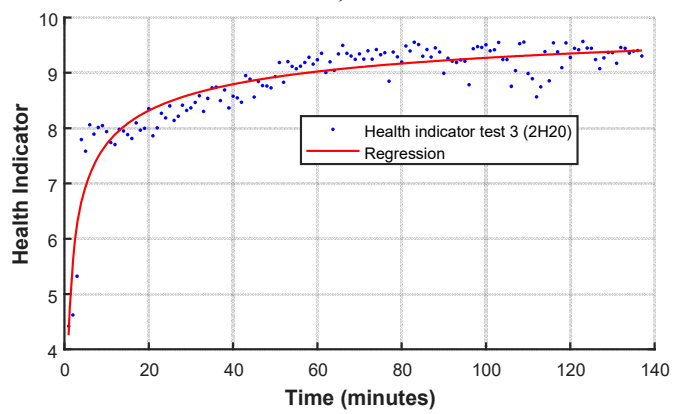

c)

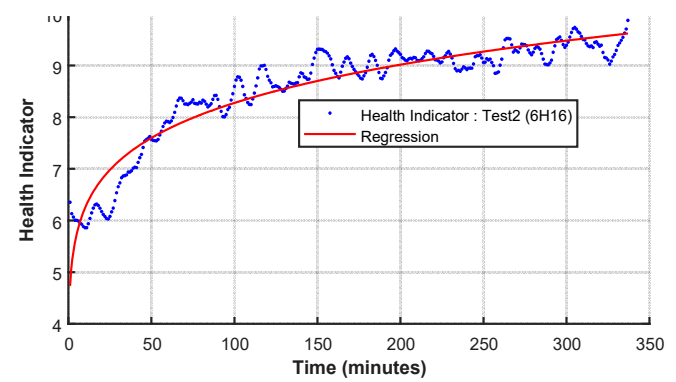

b)

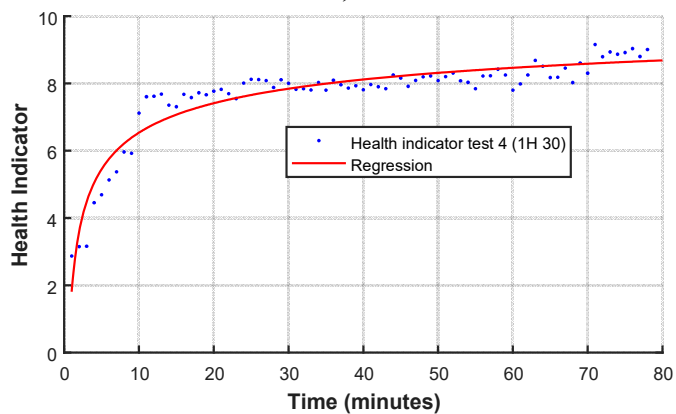

d)

Fig. 12. Health indicator for the tested bearings

From Table 5, we can find a considerable improvement in the classification accuracies compared to the results obtained by using the WPD based fault prognostics method with different loading of the classifier. Another popular metric for the performances evaluation method is the Root Mean Square Error (RMSE). The RMSE of RUL estimation is employed as a performance measure shown in Fig. 13.

From the above four experiments, we can find that the classification accuracies obtained by using the CNNs and WPD are higher than those obtained by using WPT and statistical parameters-based fault prognostics method [34].

The regression results are presented in Table 5 in terms of the factors of determination $R^{2}$ for the different training models. The $R$ values, indicating the fraction of the total variance that could be explained by the model, are very high. From the results, it is seen that all the predictors perform very well. The objective is to apply the best power fit on the degradation model obtained by Eq. (6): 
$f(t)=a \cdot t^{b}+c$,

$R U L(t)=t_{\text {final }}-H I^{-1}(t)$

where $t_{\text {final }}$ is the time when the fault occurs and $H I^{-1}$ the inverse of the health indicator $H I(t)$ used to get the current cycle or time $(t)$. The validation of these results shown in Table 5 by computing of the sum square error (SSE), $R^{2}$ RMSE.

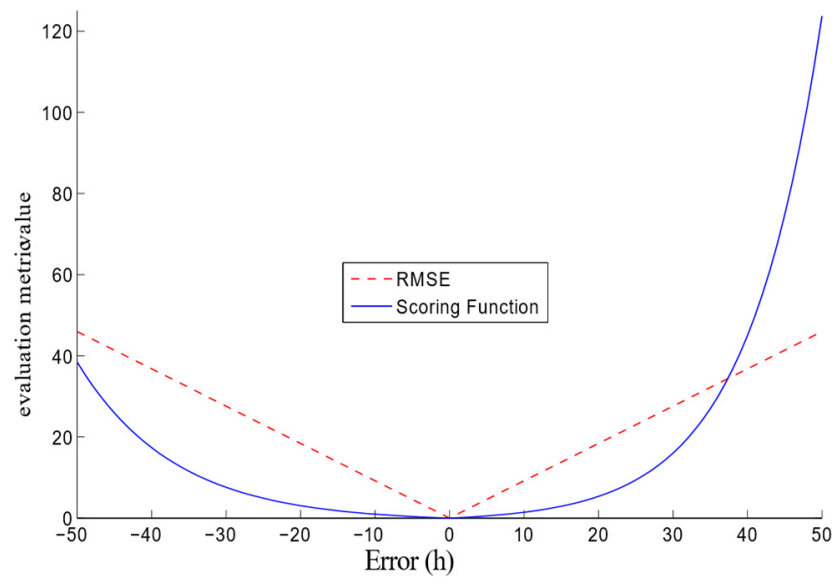

Fig. 13. Comparison between scoring function and error value

Table 5. Prediction performance

\begin{tabular}{|c|c|c|c|}
\hline Bearings & RMSE & $R^{2}$ & Regression function \\
\hline H50 & 0.2285 & 0.9451 & $f(t)=-8.058 * t^{-0.3166}+10.59$ \\
\hline H16 & 0.3019 & 0.9342 & $f(t)=-8.335 * t^{-0.3096}+10.42$ \\
\hline H20 & 0.2996 & 0.9258 & $f(t)=-6.297 * t^{-0.3458}+10.55$ \\
\hline H30 & 0.4168 & 0.8961 & $f(t)=-9.237 * t^{-0.3117}+11.04$ \\
\hline
\end{tabular}

The RUL estimation is the distance between the current time and the time for which the regression model given in Eq. (5). The threshold or the acceptable limit of the vibration magnitude [28] of each degradation in bearings, corresponds to the end of each experiment. The power fitting of the smoothed health indicator is shown in Fig. 12.

\subsection{Temperature correlation}

The correlation between temperature and health indicator given a result shown in Fig. 14. More advanced prognostics interested on performance degradation assessment, so that failures can be predicted and prevented using the temperature and vibrations signal obtained in this study by $\mathrm{CNNs}$ for regression. As soon as, the concept of correlation between the two indicators for accurately assessing the bearing performance degradation is a critical step toward realizing an online tool condition monitoring platform. The results of its application for performance degradation assessment show that this indicator can reflect effectively the performance of degradation process.

It has been shown that the health indicator obtained by vibrations measurements given the same monotonicity compared to temperature measurements. 


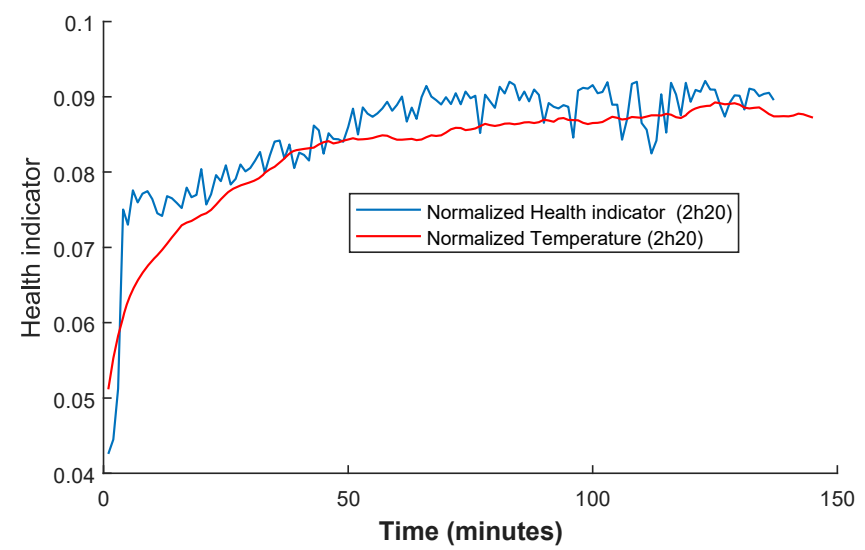

Fig. 14. Temperature and health indicator obtained by CNNs

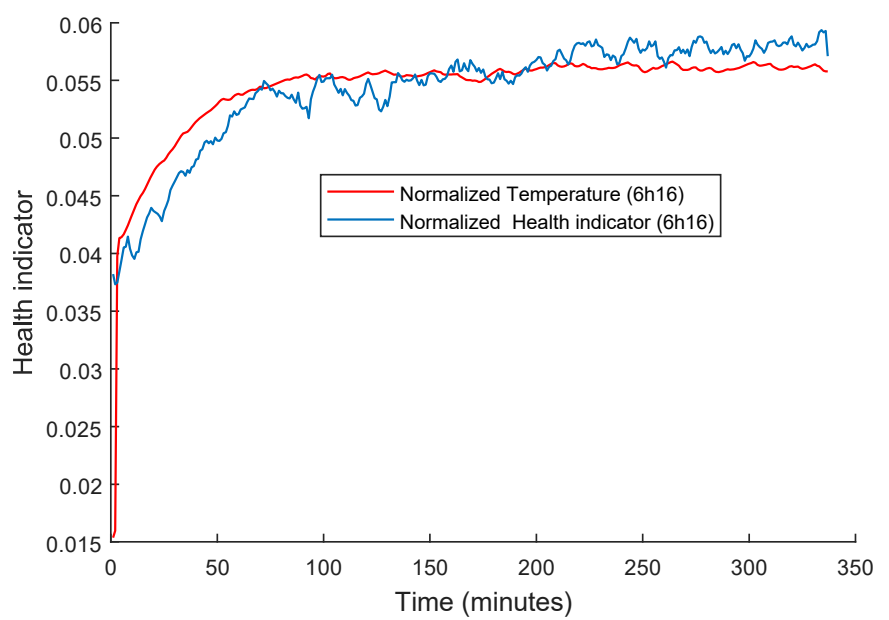

Fig. 15. Temperature and health indicator obtained by $\mathrm{CNNs}$

Dalila Belmiloud, Tarak Benkedjouh and Mohamed Lachi conceived of the presented idea for the bearings prognostics and correlation with temperature using artificial intelligence techniques. Dalila Belmiloud developed the theory of deep learning and performed the computations with the decision making of the score correlation between health indicator and temperature evolution. Mohamed Lachi and Ali Laggoun verified the analytical methods from the different stages of looping and convolution. Tarak Benkedjouh encouraged Dalila Belmiloud to investigate and supervised the findings of this work. All authors discussed the results and contributed to the final manuscript and the different steps for writing the documents. Dalila Belmiloud developed the theoretical formalism, performed the analytic calculations and performed the numerical simulations from experimental data. Both Dalila Belmiloud and Tarak Benkedjouh authors contributed to the final version of the manuscript. Tarak Benkedjouh supervised the project. Dalila Belmiloud, Tarak Benkedjouh, Mohamed Lachi, Ali Laggoun, Jean Paul Dron conceived and planned the experiments and contributed to sample preparation and the interpretation of the results. Dalila Belmiloud took the lead in writing the manuscript. All authors provided critical feedback and helped shape the research, analysis and manuscript. Dalila Belmiloud and Tarak Benkedjouh designed the model and the computational framework and analyzed the data from data acquisition, features extraction and remaining useful life estimation. Dalila Belmiloud and Mohamed Lachi carried out the implementation and wrote the manuscript with input from all authors. Dalila Belmiloud designed and derived the models and analyzed the data. Tarak Benkedjouh assisted all 
measurements from PRONOSTIA experimental setup. Dalila Belmiloud and Tarak Benkedjouh worked out almost all of the technical details, and performed the numerical implementation of the suggested experiment. Dalila Belmiloud, Tarak Benkedjouh, Mohamed Lachi and Ali Laggoun contributed to the design and implementation of the research with future scope and listing the efficiency when compared with other methods and the future scope of the work implemented on this paper and the limitation of the proposed solution in practice, to the analysis of the results and to the writing of the manuscript.

\section{Conclusions}

In this paper, a novel fault prognostics method for REBs based on WPD and CNNs have been proposed. The model develops an effective RUL prediction method that addresses multiple challenges in complex system prognostics where many parameters are unknown. In order to investigate the effectiveness for practical applications, the proposed method is tested by using PRONOSTIA experimental setup. The test results show that the proposed method can achieve a higher classification accuracy rate than the result obtained by using wavelet packet transform based prognostics method. The good experimental results obtained method, further architecture optimization is still necessary. Deep learning methods generally suffer from high computing load, and that will be focused on in further research. In this study Temperature and vibration analysis are two dominating condition monitoring techniques applied to bearing failures prognostics. The temperature HI has reliable revealed the all change of the bearings condition monitoring and rendering the diagnosis procedure less complicated. For the proposed algorithm evaluation, we examined the performance on four bearing's data sets. The experimental results of bearing data sets demonstrate the superiority of the proposed CNNs model to other fault-prognostics methods. The proposed approach achieves a high degree of accuracy.

\section{References}

[1] Condition Monitoring and Diagnostics of Machines - Prognostics - Part 1: General Guidelines. NF ISO 13381-1, 2005.

[2] Jaouher Ben Ali, Brigitte Chebel Morello, Lotfi Saidi, Simon Malinowski, Farhat Fnaiech. Accurate bearing remaining useful life prediction based on Weibull distribution and artificial neural network. Mechanical Systems and Signal Processing, Vol. 56, 2015, p. 150-172.

[3] Dario Amodei, Sundaram Ananthanarayanan, Rishita Anubhai, Jingliang Bai, Eric Battenberg, Carl Case, Jared Casper, Bryan Catanzaro, Qiang Cheng, Guoliang Chen, et al. Deep speech 2: End-to-end speech recognition in English and Mandarin. International Conference on Machine Learning, 2016, p. 173-182.

[4] Giduthuri Sateesh Babu, Zhao Peilin, Li Xiao Li Deep convolutional neural network based regression approach for estimation of remaining useful life. International Conference on Database Systems for Advanced Applications, 2016, p. 214-228.

[5] Cerrada Mariela, Sánchez René Vinicio, Li Chuan, Pacheco Fannia, Cabrera Diego, Oliveira José Valente De, Vásquez Rafael E. A review on data-driven fault severity assessment in rolling bearings. Mechanical Systems and Signal Processing, Vol. 99, 2018, p. 169-196.

[6] Li Deng. A tutorial survey of architectures, algorithms, and applications for deep learning. APSIPA Transactions on Signal and Information Processing, Vol. 3, 2014, https://doi.org/10.1017/atsip.2013.9.

[7] Gebraeel Nagi, Lawley Mark, Liu Richard, Parmeshwaran Vijay. Residual life predictions from vibration-based degradation signals: a neural network approach. IEEE Transactions on Industrial Electronics, Vol. 51, Issue 3, 2004, p. 694-700.

[8] Goyal D., Pabla B. S. The vibration monitoring methods and signal processing techniques for structural health monitoring: a review. Archives of Computational Methods in Engineering, Vol. 23, Issue 4, 2016, p. 585-594.

[9] Guo Liang, Li Naipeng, Jia Feng, Lei Yaguo, Lin Jing. A recurrent neural network based health indicator for remaining useful life prediction of bearings. Neurocomputing, Vol. 240, 2017, p. 98-109.

[10] Guo Xiaojie, Chen Liang, Shen Changqing. Hierarchical adaptive deep convolution neural network and its application to bearing fault diagnosis. Measurement, Vol. 93, 2016, p. 490-502. 
[11] Yen G. G., Lin Kuo Chung. Wavelet packet feature extraction for vibration monitoring. IEEE Transactions on Industrial Electronics, Vol. 47, Issue 3, 2000, p. 650-667.

[12] He Kaiming, Zhang Xiangyu, Ren Shaoqing, Sun Jian. Deep residual learning for image recognition. Proceedings of the IEEE Conference on Computer Vision and Pattern Recognition, 2016, p. $770-778$.

[13] Javed Kamran, Gouriveau Rafael, Zerhouni Noureddine. State of the art and taxonomy of prognostics approaches, trends of prognostics applications and open issues towards maturity at different technology readiness levels. Mechanical Systems and Signal Processing, Vol. 94, 2017, p. 214-236.

[14] Jia Feng, Lei Yaguo, Lin Jing, Zhou Xin, Lu Na. Deep neural networks: a promising tool for fault characteristic mining and intelligent diagnosis of rotating machinery with massive data. Mechanical Systems and Signal Processing, Vol. 72, 2016, p. 303-315.

[15] Jing Luyang, Zhao Ming, Li Pin, Xu Xiaoqiang. A convolutional neural network based feature learning and fault diagnosis method for the condition monitoring of gearbox. Measurement, Vol. 111, 2017, p. 1-10.

[16] Khelif Racha, Morello Brigitte Chebel, Malinowski Simon, Laajili Emna, Fnaiech Farhat, Zerhouni Noureddine. Direct remaining useful life estimation based on support vector regression. IEEE Transactions on Industrial Electronics, Vol. 64, Issue 3, 2017, p. 2276-2285.

[17] Santosh Kumar, Amit Pandey, Sai Ram Satwik K., Sunil Kumar, Sanjay Kumar Singh, Amit Kumar Singh, Anand Mohan. Deep learning framework for recognition of cattle using muzzle point image pattern. Measurement, Vol. 116, 2018, p. 1-17.

[18] Lecun Yann, Boser Bernhard E., Denker John S., Henderson Donnie, Howard Richard E., Hubbard Wayne E., Jackel Lawrence D. Handwritten digit recognition with a back-propagation network. Advances in Neural Information Processing Systems, 1990, p. 396-404.

[19] Lei Yaguo, Li Naipeng, Guo Liang, Li Ningbo, Yan Tao, Lin Jing. Machinery health prognostics: A systematic review from data acquisition to RUL prediction. Mechanical Systems and Signal Processing, Vol. 104, 2018, p. 799-834.

[20] Li Xiang, Ding Qian, Sun Jian Qiao. Remaining useful life estimation in prognostics using deep convolution neural networks. Reliability Engineering and System Safety, Vol. 172, 2018, p. 1-11.

[21] Li Zhixiong, Jiang Yu, Guo Qiang, Hu Chao, Peng Zhongxiao. Multi-dimensional variational mode decomposition for bearing-crack detection in wind turbines with large driving-speed variations. Renewable Energy, Vol. 116, 2018, p. 55-73.

[22] Nectoux Patrick, Gouriveau Rafael, Medjaher Kamal, Ramasso Emmanuel, Morello Brigitte Chebel, Zerhouni Noureddine, Varnier Christophe. Pronostia: an experimental platform for bearings accelerated degradation tests. IEEE International Conference on Prognostics and Health Management, 2012.

[23] Pietrini Giorgio, Soldati Alessandro, Barater Davide, Concari Carlo. Wavelet-based prognosticoriented temperature sensing with sigma-delta ADCS in power applications. Energy Conversion Congress and Exposition (ECCE), 2017, p. 1465-1472.

[24] Akhand Rai, Upadhyay S. H. The use of MD-CUMSUM and NARX neural network for anticipating the remaining useful life of bearings. Measurement, Vol. 111, 2017, p. 397-410.

[25] Safizadeh M. S., Mohammadi S., Nouri N. M. Using vibration signals for cavitation monitoring in centrifugal pumps. Aerospace Mechanics, Journal Vol. 10, Issue 3, 2014, p. 25-34.

[26] Schmidhuber Jürgen. Deep learning in neural networks: an overview. Neural Networks, Vol. 61, 2015, p. 85-117.

[27] Sun Wenjun, Shao Siyu, Zhao Rui, Yan Ruqiang, Zhang Xingwu, Chen Xuefeng. A sparse autoencoder-based deep neural network approach for induction motor faults classification. Measurement, Vol. 89, 2016, p. 171-178.

[28] Tamilselvan Prasanna, Wang Yibin, Wang Pingfeng. Deep belief network based state classification for structural health diagnosis. Aerospace Conference, 2012.

[29] Wang Dong, Tsui Kwok Leung, Miao Qiang. Prognostics and health management: a review of vibration based bearing and gear health indicators. IEEE Access, Vol. 6, 2018, p. 665-676.

[30] Wang Yanxue, Xiang Jiawei, Markert Richard, Liang Ming. Spectral kurtosis for fault detection, diagnosis and prognostics of rotating machines: a review with applications. Mechanical Systems and Signal Processing, Vol. 66, 2016, p. 679-698. 
[31] Yuan Mei, Wu Yuting, Lin Li. Fault diagnosis and remaining useful life estimation of aero engine using lstm neural network. IEEE International Conference on Aircraft Utility Systems, 2016, p. 135-140.

[32] Yu Jianbo. Adaptive hidden Markov model-based online learning framework for bearing faulty detection and performance degradation monitoring. Mechanical Systems and Signal Processing, Vol. 83, 2017, p. 149-162.

[33] Zhao Rui, Yan Ruqiang, Chen Zhenghua, Mao Kezhi, Wang Peng, Gao Robert X. Deep learning and its applications to machine health monitoring: A survey. arXiv preprint arXiv:1612.07640, 2016.

[34] Peng Z. K., Chu F. L. Application of wavelet transform in machine condition monitoring and fault diagnostics: a review with bibliography. Mechanical System and Signal Processing, Vol. 18, Issue 2, 2004, p. 199-221.
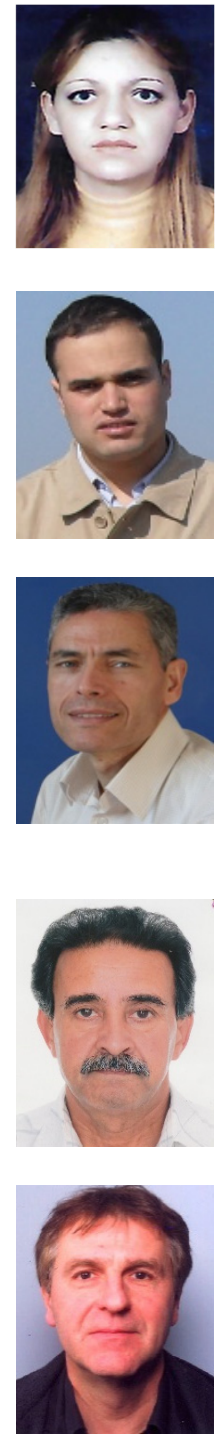

Dalila Belmiloud received her higher study diploma in physics degree from USTHB University in 2002, then a postgraduate degree in solid physics with high honors from the of Algiers University USTHB in 2004. Ph.D. student between the University of Reims Champagne Ardennes URCA and Mhamed Bouguera University UMBB. Her research focuses on the theory and applications of statistical signal processing, in particular nonstationary signal analysis for bearing failure analysis. She is interested in developing methods for efficient signal representation, detection and classification.

Tarak Benkedjouh received his engineering degree from National Engineers and Technicians School of Algiers (ENITA) in 2004. He received his Ph.D. degree in mechanical engineering from the Polytechnic School in 2013. Currently he is working at EMP and AS2M department in FEMTO-ST Institute (France), as researcher in PHM group. His research activities are concerned with condition monitoring and prognostics of mechanical components and structural health monitoring. His main research activities are concerned with intelligent maintenance systems, and e-maintenance.

Mohammed Lachi received his engineering degree from Algerian Petroleum Institute of Boumerdes (IAP). He received his Ph.D. in mechanics and energetic from the National Polytechnic Institute of Lorraine (INPL) in 1991. He then got his Habilitation to direct research, HDR, from the University of Reims Champagne Ardenne in 2002. In September 1992, he joined the University of Reims Champagne Ardennes. His current research interests in GRESPI laboratory include heat exchangers, conjugate heat transfer, development of thermomechanical behavior of materials, heat transfer in mechanical systems

Ali Laggoun received his engineering degree from the University of Algiers in 1978 with a postgraduate degree in solid physics. After that, he started a teaching career at the University of Boumerdes in 1981. He prepared a Ph.D. in materials science at the University of Rouen (France) from 1987 to 1992 . Back in Algeria, he started a new career as a teacher-researcher in the field of materials sciences, as Associated Professor, then as a Professor in the UR-MPE research unit of the University of Boumerdes. He done more than 30 international publications and participated in several master's and Ph.D. programs.

Jean-Paul Dron received his engineering degree from University of Art Et Métier Paris in 1993. He received his Ph.D. degree in 1995 from the University of Reims URCA and he joined the University of Reims Champagne Ardennes as a Professor. He then got his Habilitation to direct research, HDR, from the University of Reims Champagne Ardenne in 2000. As for investigation, he is with GRESPI of the application of vibration and angular analysis in the conditional and predictive maintenance of systems operating in stationary and non-stationary regimes under different operating conditions. His research interests include the design and control of rotating machinery faults detection and failure analysis, finite-element methods for mechanical behavior, and fault prognosis and mitigation of electrical drive systems. 